
\title{
25 Research Sourere \\ Exploring the Contexts, Mechanisms and Outcomes of a Dental Anxiety Service in Norway: A Realist Evaluation
}

Emilie Bryne ( $\nabla$ emilie.bryne@throg.no )

Social Sciences https://orcid.org/0000-0001-9533-1862

Sarah Catherine Patricia Duff Hean

University of Stavanger: Universitetet i Stavanger

Kjersti Berge Evensen

Oral Health Centre of Expertise Rogaland Norway

Vibeke Hervik Bull

Oral Health Centre of Expertise Rogaland Norway

Research article

Keywords: dental anxiety, dental phobia, torture, abuse, oral health, dental health services, health services, oral health policy

Posted Date: March 2nd, 2021

DOI: https://doi.org/10.21203/rs.3.rs-279468/v1

License: (c) (i) This work is licensed under a Creative Commons Attribution 4.0 International License. Read Full License 


\section{Abstract}

Background: Torture, abuse and dental phobia are often precursors to developing a pathological relationship with dental care due to elevated anxiety and the risk for re-traumatisation. Consequently, this patient group tends to avoid dental services, leaving them with severe tooth decay that affects both their general and psychosocial health.

Norwegian dental services have implemented a specific dental service targeting this patient group, aiming to both alleviate their dental anxiety and restore their oral health. The outcomes of this service have been positive, but for this model to be transferrable to other national contexts, it is necessary not just to understand whether the service works, but also how and why it works. Therefore, this study developed theories on how the structure of the service alleviates dental anxiety and restores patients' oral health. Although developed specifically in a Norwegian context, these theories may be applicable to other national and international contexts.

Methods: This realist evaluation comprised sequential, multiple methods encompassing a review of service and policy documentation $(n=12)$, followed by realist interviews with service developers and deliverers $(n=12)$. Guided by a retroductive approach consisting of coding, cataloguing and configuring through content analyses and context-mechanism-outcome (CMO) heuristics, the analyses generated four programme theories.

Results: First, the state-subsidised dental service affects service access and service uptake. Second, this service can be adapted and tailored to regional resources to meet the needs of the heterogenous patient group. Third, regional service teams are cohesive because of a lack of national communication and cooperative practice. Fourth, the complexity of migration processes and poor dissemination practices leads to poor recruitment of torture survivors to the service.

Conclusions: The service follows a hybrid bottom-up, top-down approach, allowing teams to practise discretion and tailor their approach to meet individual needs. With its bi-dimensional structure, the service reaches a patient population that would otherwise avoid dental services. Service uptake is beneficial as patients report experiencing improved quality of life. However, the service is struggling to reach torture survivors, which may be attributable to multiple contextual factors. More research is therefore required to understand the lack of service uptake among torture survivors.

\section{Background}

People subjected to torture or abuse or who suffer from dental phobia often avoid dental services and examinations because they may trigger re-traumatisation or elevated anxiety [1-3]. Ongoing avoidance of dental services, stemming from the fear of 'revealing' the mouth, leads to deteriorated oral health, which often requires acute dental relief. This can be both painful and costly not just for individuals (e.g. impaired social and work opportunities, often leading to social isolation) but for society as a whole [4-7]. 


\section{The Tada Service}

Despite the realities of international immigration and the well-recognised plight of asylum seekers, especially survivors of torture or abuse, as well as the prevalence of dental phobia among patients, our review of the literature found no evidence of dental services actively addressing the oral health and anxiety needs of these vulnerable patient groups. We therefore highlight the uniqueness of the bidimensional approach of the TADA (torture, abuse and dental anxiety) service, which targets both anxiety and avoidance of dental treatment among patients who have survived abuse or torture or who suffer from dental phobia for the purpose of restoring and maintaining their dental health.

The TADA service was developed in 2010 by the Norwegian government to address the challenges faced by patients who have survived torture or abuse or who suffer from dental phobia in receiving dental services [8]. A specific working group was established to design and implement a dental service catering specifically to these patients $[8,9]$. The working group is a joint venture between dental practitioners from the private and public sector, psychologists specialising in anxiety treatment, and researchers from the field of dentistry. Evidence from national and international research suggests that elements of cognitive behavioural therapy (CBT), especially exposure therapy, could serve as an effective treatment for anxiety, which is assumed to be a precursor to avoidance behaviour [10-12]. The working group, therefore, determined that CBT could be key to facilitating effective treatment outcomes via the TADA service. The underlying assumption in this regard is that by relieving dental anxiety among vulnerable patients, their oral health could be restored.

Since early 2013, the TADA service has been gradually implemented across Norway [8] as a public service - it is free for TADA patients. Subsidising the service is considered essential, as otherwise the fees typically associated with dental services could prevent access to dental examinations by TADA patients $[13,14]$. Currently, 52 TADA teams are in place across Norway. According to the TADA service, as of 2018, 1186 patients have either completed or are undergoing dental treatment.

The TADA service is in high demand, as reflected in the national waiting list, which is a year long. Patients eligible to receive this service are those whose ability to care for their dental or general health has been adversely impacted by, in the first instance, a past history of torture with direct consequences for their mouth, neck or head regions. Torture survivors often display comorbidities and are likely struggling with post-traumatic stress disorder (PTSD). As of 2019, approximately 10,000-35,000 torture survivors were residing in Norway [15].

The second patient group eligible for the TADA service are those whose dental behaviours have been adversely impacted by sexual, physical or psychological abuse. Norwegian national reports have revealed that 34 per cent of women and 11 per cent of men have been subjected to sexual abuse, and that one in 20 children and adolescents are assaulted during their childhood years $[16,17]$. The third eligible patient group are those who meet the Diagnostic and Statistical Manual of Mental Disorders-IV criteria for suffering from a specific phobia, which is defined as an unreasonable fear in the presence, or in the 
anticipated presence, of a feared object, eliciting a pathological response [18]. To the best of our knowledge, no studies have determined the national prevalence of dental phobia. However, a recent study from northern Norway (Troms $\varnothing$ ) revealed that 8 per cent of 18-year-olds suffer from dental phobia [19].

The TADA service aims to deliver an equitable, standardised service to adults ( $>18$ years) across Norway. However, oral health in the nation is administered at the county level, meaning that each county is responsible for the logistics of service and resource provisions. Meanwhile, the long waiting list and increased implications of poor oral health for torture and abuse survivors [20-28] have prompted a yearly budget increase for the TADA service, increasing from 2.5 million kroners in 2011 to 85 million kroners (equivalent to 8 million euros) in 2020.

From a national perspective, the TADA service is considered successful, having managed to enrol 1186 patients exhibiting avoidance behaviour in 2018. That said, the exact nature of the relationship between government investment in the TADA service, the implementation and delivery of the service, and service outcomes remains poorly understood. How, for example, is the TADA service structured to cater to such a heterogeneous patient group? And why is its bi-dimensional service programme successful for these patients $[10-12,29,30]$ ?

The current study addressed these questions by conducting a realist evaluation of how the TADA service works, for whom, under which circumstances, with what resources, and why. The evaluation included an exploration of the contextual elements, triggering mechanisms and resulting outcomes of the TADA service. Findings related to the role of dental practitioners in delivering the service and the service's impact on patients are reported elsewhere (paper in review).

We argue that it is of international interest to learn how the TADA service is structured to target its contextual and vulnerable patient group given the service's actual and potential societal and individual benefits. Such knowledge can also contribute to the broader field of dental anxiety treatment and dental health services for patients with a history of torture or abuse or a diagnosis of dental phobia.

\section{Methods}

\section{A realist evaluation methodology}

To explore the structure and functions of the TADA service and thereby expand knowledge on dental anxiety treatments and dental health services for vulnerable groups, a realist evaluation methodology was employed [31]. Intrinsic to this methodology was the exploration of for whom and under which circumstances the TADA service is effective as well as the assessment of its complex programmes and services [32,33]. This complexity is inherent to the service's interdisciplinary approach and diverse patient population. A realist evaluation is well suited in this regard as it allows the researcher to more fully describe the working mechanisms of complex systems - and indeed, realist evaluations are becoming increasingly popular for addressing the complexities of health services [31, 33, 34]. Realist evaluations are underpinned by the philosophy of scientific realism, which claims that generative causal mechanisms 
are triggered by contextual elements that, in turn, produce observable outcomes $[31,33,35,36]$. It is this philosophy that distinguishes realist evaluations from other types of theory-driven approaches, insofar as the realist endeavour generates data-derived insights at an ontological depth that permits valid explanations for why a given phenomenon works, for whom, and under which circumstances [31, 35].

A realist evaluation begins by seeking to explain how a phenomenon (e.g. TADA programme activities) contributes to substantive changes (e.g. treatment outcomes) [37, 38]. Underlying the realist evaluation in the current study was the assumption that the way in which the service was developed was guided by one or more theories and that service developers either implicitly or explicitly rely on these theories when further developing the service in specific contexts and circumstances [31]. A key goal of the realist evaluation is therefore to articulate programme theories - namely, to provide plausible explanations for the nature and characteristics of a phenomenon by describing its outcomes, the mechanisms responsible for these outcomes, and how these outcomes are triggered in a given contextual setting. Table 1 presents more precise definitions of outcomes, mechanisms and contexts, in reverse order.

Table 1

Definitions and Reflections on Contexts, Mechanisms and Outcomes [39]

Contexts: These describe the elements and background factors that allow mechanisms to be triggered. Contexts are not limited to locations but also refer to characteristics of individuals (e.g. in the present study, to characteristics of service developers, deliverers and patients) and to interrelationships between actors and institutional settings and their placement within wider infrastructural settings. To understand contexts, the researcher should ask what conditional elements or contextual components need to be present for a mechanism to be triggered?

Mechanisms: In realist evaluations, it is believed that if the context is conducive, then a mechanism will be triggered. This conception of mechanisms has two main features: resources and reasoning. The assumption here is that if certain service resources are introduced to a specific context, they will generate changes in the actors' reasoning. In this study, these actors can be service developers, deliverers or patients. Pairing resources with reasoning defines the mechanism. The following question should therefore be asked to reveal mechanisms: how do the resources provided by the service impact the service deliverers and on what assumptions, values and beliefs do service users rely when interacting with these resources? Furthermore, what is being triggered in the service, and to what particular outcome does it lead?

Outcomes: Outcomes describe to what visible output or impacts the mechanisms lead. These outcomes can be immediate, intermediate or long term. Therefore, in analysing outcomes, the researcher should ask to what does the triggered mechanism lead, and what are the resulting outcomes of mechanisms triggered in the right context?

Context-mechanism-outcome (CMO) configurations: By heuristically configuring contexts, mechanisms and outcomes, generative explanations for the observed outcomes can be produced. These $\mathrm{CMO}$ configurations provide a causative explanation for the entire service or for specific features of the service, or they are embedded in another $\mathrm{CMO}$ configuration developed for the same purpose.

The realist evaluation method deconstructs a phenomenon (in this study, the TADA service) in terms of contexts, mechanisms and outcomes. It then reconstructs these factors in a series of CMOCs (contextmechanism-outcome configurations). It is this configuration of contexts, mechanisms and outcomes that yields a proposition about how the phenomenon works, for whom, and under which circumstances [31, $34,40]$. In principle, the process involves making foundational assumptions about how the phenomenon 
(i.e. service/programme) should work, explicitly, before systematically collecting evidence to test and refine or refute this theory $[31,34,40]$. Consequently, and circularly, the evaluation process starts with a theory and culminates with a theory. The whole process is by nature iterative and retroductive, aiming to elicit the underlying mechanisms believed to reside at an ontological depth.

\section{Data Collection}

The first author immersed herself in the research context by participating in regional and national TADA service meetings and network gatherings and by shadowing TADA practitioners. Throughout this work, she made memos from meetings and maintained a reflexive journal. The first author had master-level qualitative interviews experience and training.

This study employed a multi-method study design to generate the programme theories with two primary data sources: service and policy documents, and interviews [41-43]. While typically, existing literature in the field can be reviewed and synthesised to contribute to these theories (33), in the present study, a literature search on dental services aimed at TADA patients was unproductive, thereby underscoring the apparent uniqueness of the research topic. Accordingly, interviews combined with service and policy documents were the sole data sources used.

The Norwegian Centre for Research Data approved the research protocol.

Document analysis

A search of service databases and interviews with stakeholders generated 12 policy documents (Table 2) and service-related grey literature. Reviewing these documents yielded a historical picture and understanding of how the TADA service operates and intends to achieve its objectives, as well as descriptions of the service's elements, including the desired therapy (CBT). Consistent with sequential, multi-method design [44], the analysis of the policy documents preceded the interviews and thereby informed the structure and foci of the interview schedule. 
Table 2

Document Analyses

\begin{tabular}{|c|c|c|c|c|}
\hline $\mathrm{Nr}$ & $\begin{array}{l}\text { Title (translated } \\
\text { into English): }\end{array}$ & Author/Year & $\begin{array}{l}\text { Document } \\
\text { type }\end{array}$ & Description \\
\hline 1 & $\begin{array}{l}\text { Practitioners } \\
\text { Handbook }\end{array}$ & $\begin{array}{l}\text { Myran, L., } \\
\text { Johnsen, } \\
\text { I.B., Arøen } \\
\text { Lie, J.P., } \\
\text { June } 2019\end{array}$ & Handbook & $\begin{array}{l}\text { This handbook provides details on how } \\
\text { practitioners should meet and work with the } \\
\text { contextual patient group. Details regarding } \\
\text { the aetiology of anxiety, symptoms of dental } \\
\text { phobia, cognitive behavioural therapy and } \\
\text { communication methods aimed at enhancing } \\
\text { relationship building are elaborated in this } \\
\text { handbook. }\end{array}$ \\
\hline 2 & $\begin{array}{l}\text { Practitioners } \\
\text { Guidance }\end{array}$ & $\begin{array}{l}\text { December } \\
2018\end{array}$ & $\begin{array}{l}\text { Guidelines } \\
\text { on the } \\
\text { operating } \\
\text { practice }\end{array}$ & $\begin{array}{l}\text { This guidance leaflet describes some } \\
\text { potential service routes for the patient, } \\
\text { resources (such as templates for anxiety } \\
\text { treatment), inclusion and exclusion criteria for } \\
\text { patients, and overall aspects practitioners } \\
\text { should consider (such as collegial support } \\
\text { and collaborating with others). }\end{array}$ \\
\hline 3 & $\begin{array}{l}\text { Treatment } \\
\text { contract and } \\
\text { TADA info }\end{array}$ & N/A & Service aid & $\begin{array}{l}\text { The treatment contract supports joint } \\
\text { relationships and collaborative work in } \\
\text { restoring the oral health of patients. }\end{array}$ \\
\hline 4 & Treatment plan & N/A & Service aid & $\begin{array}{l}\text { The treatment plan is a template and outline } \\
\text { for each session and describes the small and } \\
\text { large goals intended for the patient to achieve } \\
\text { throughout the service pathway. }\end{array}$ \\
\hline 5 & Coping plan & N/A & Service aid & $\begin{array}{l}\text { This coping plan is jointly completed by the } \\
\text { patient and a TADA dental practitioner. The } \\
\text { coping plan aims to aid in the dental } \\
\text { restoration phase, making the patient and the } \\
\text { follow-up dental practitioner aware of their } \\
\text { anxiety triggers, warnings and the need for } \\
\text { adjustment. }\end{array}$ \\
\hline 6 & $\begin{array}{l}\text { Patient } \\
\text { handbook }\end{array}$ & 2019 & Guidebook & $\begin{array}{l}\text { Patients receive a handbook describing the } \\
\text { aim and outline of the service. The handbook } \\
\text { includes details on anxiety and trauma and } \\
\text { the effects they have on the dental setting. }\end{array}$ \\
\hline \multirow[t]{2}{*}{7} & White Paper 35 & \multirow[t]{2}{*}{$2006-2007$} & \multirow{2}{*}{$\begin{array}{l}\text { Policy } \\
\text { paper }\end{array}$} & \multirow{2}{*}{$\begin{array}{l}\text { Describes the government's objective to } \\
\text { create and offer equal health care services } \\
\text { regardless of diagnosis, place of residence, } \\
\text { personal finances, gender, ethnic background } \\
\text { and individual life circumstances. }\end{array}$} \\
\hline & $\begin{array}{l}\text { Accessibility, } \\
\text { expertise and } \\
\text { social } \\
\text { equalisation in } \\
\text { the future dental } \\
\text { health service }\end{array}$ & & & \\
\hline
\end{tabular}




\begin{tabular}{|c|c|c|c|c|}
\hline $\mathrm{Nr}$ & $\begin{array}{l}\text { Title (translated } \\
\text { into English): }\end{array}$ & Author/Year & $\begin{array}{l}\text { Document } \\
\text { type }\end{array}$ & Description \\
\hline 8 & $\begin{array}{l}\text { Facilitated } \\
\text { dental health } \\
\text { services for } \\
\text { people who } \\
\text { have been } \\
\text { subjected to } \\
\text { torture, abuse or } \\
\text { odontophobia }\end{array}$ & $\begin{array}{l}\text { The } \\
\text { Norwegian } \\
\text { Directorate } \\
\text { of Health, } \\
\text { October } \\
2010\end{array}$ & Report & $\begin{array}{l}\text { The first report developed prior to TADA } \\
\text { teams being established. This report gives a } \\
\text { description of different aspects of the } \\
\text { patients, as well as the rationale for why they } \\
\text { need facilitated dental treatment or therapy. }\end{array}$ \\
\hline 9 & $\begin{array}{l}\text { Job description: } \\
\text { Dentist/Dental } \\
\text { Hygienist }\end{array}$ & $\mathrm{N} / \mathrm{A}$ & $\begin{array}{l}\text { Role } \\
\text { description }\end{array}$ & $\begin{array}{l}\text { This job description describes the expected } \\
\text { tasks the dental practitioner should execute. }\end{array}$ \\
\hline 10 & $\begin{array}{l}\text { Job description: } \\
\text { Dental Assistant }\end{array}$ & N/A & $\begin{array}{l}\text { Role } \\
\text { description }\end{array}$ & $\begin{array}{l}\text { This job description describes the expected } \\
\text { tasks the dental assistant should execute. }\end{array}$ \\
\hline 11 & $\begin{array}{l}\text { Job description: } \\
\text { Psychologist }\end{array}$ & $\mathrm{N} / \mathrm{A}$ & $\begin{array}{l}\text { Role } \\
\text { description }\end{array}$ & $\begin{array}{l}\text { This job description describes the expected } \\
\text { tasks the psychologist should execute. }\end{array}$ \\
\hline 12 & TADA survey & $\begin{array}{l}\text { Simonsen, } \\
\varnothing ., 2019\end{array}$ & Survey & $\begin{array}{l}\text { This is a survey conducted by a private } \\
\text { dentist (not a TADA service practitioner) that } \\
\text { collected thoughts from other (mostly private) } \\
\text { practitioners regarding the TADA service. } \\
\text { Thirty statements were reported, all of which } \\
\text { voiced negative concerns about the workings } \\
\text { of the service. }\end{array}$ \\
\hline 13 & $\begin{array}{l}\text { Overall reporting } \\
\text { on the TADA } \\
\text { service }\end{array}$ & $\begin{array}{l}\text { The } \\
\text { Norwegian } \\
\text { Directorate } \\
\text { of Health, } \\
2016,2017 \\
2018,2019\end{array}$ & Report & $\begin{array}{l}\text { Yearly reporting of data on the types of } \\
\text { patients enrolled in the service, waiting lists, } \\
\text { the total number of TADA teams within each } \\
\text { county, and the economy of the service. }\end{array}$ \\
\hline
\end{tabular}

Realist interviews

The sampled population comprised professionals responsible for the development of the TADA service, nationally and regionally. That said, the TADA service is a stakeholder-driven intervention, meaning that the service developers who initially acknowledged the targeted patient population also participated in designing and implementing the service and had various roles in its delivery.

First, key informants were identified in the main service document (see Row 8 in Table 2) [8] and recruited purposively based on their role, i.e. their primary involvement in and responsibility for the initial design of the service. As the odontological field within Norway is relatively small, further recruitment occurred through a snowballing strategy $[45,46]$. Participants were recruited through email.

A total of 14 informants were identified as primary stakeholders who had a direct impact on the TADA service design and rationale. Of these informants, two has retired and 12 agreed to participate in the interviews; as two participated in the same interview, a total of 11 interviews were ultimately conducted. As mentioned above, the TADA service is stakeholder-led and implemented, meaning that the recruited 
informants played key roles in identifying the problem, conceiving and implementing the service, and acting as service deliverers. At the time the interviews took place, 10 of the 12 informants were acting as service deliverers, as either dental practitioners or psychologists. The remaining two stakeholders were members of the dentistry profession but were currently acting as managerial staff. Their role was to oversee the national structure of the TADA service as well as to develop national service guidelines and cultivate cross-sectional learning. Each of the 12 informants represented a regional manifestation of the TADA service in Norway.

All interviews were audiotaped and transcribed verbatim immediately after they were finished. Stakeholders chose to proceed interviews at their own clinic or workplace. None other than the lead author was present during the interview. The average duration of the interviews was 54 minutes. Consistent with the realist perspective, preliminary theories that emerged from the document analysis served as the basis for the initial interviews, the content of which was then used to structure subsequent interviews. Generally speaking, realist interviews have the explanatory purpose of identifying, exploring and refining emergent theories regarding the workings of a given phenomenon - in this case, the TADA service/programme [47]. TADA stakeholders were thus asked to answer questions related to their experience with and rationale for conceiving, designing, structuring, and delivering the TADA service. The interviews were semi-structured, i.e. an interview schedule was used to ensure topical consistency while, at the same time, sufficient latitude was allowed for the probing of emergent topics. Questions from the interview schedule were unique to this study and have not been published elsewhere (see supplementary file).

\section{Analyses And Data Management}

The unit of analysis in realist evaluations are programme theories [33, 39]. Programme theories comprise a set of assumptions about how, for whom, and under which circumstances a specific programme outcome occurs. More specifically, programme theories build on configurations of contexts, mechanisms and outcomes as generated from specific analytical steps, which are described below.

\section{Coding}

The first step in the analysis involves open coding. The coding procedure consists of reading the data material multiple times to ensure comprehensive understanding before assigning a code name to each fraction of the text, with each code corresponding to some aspect of the data - e.g. costly public service.

\section{Cataloguing}

Codes are then reviewed for the purpose of relating them to an area of insight. Once the codes have been related to insights concerning contexts, mechanisms or outcomes (in isolation) with respect to the CMO configuration as a unit, they are definitively catalogued. For example, in the present study, the code costly public service was catalogued under context (see Fig. 1). 


\section{Configuring}

Understanding the causal links between contexts, mechanisms and outcomes is central to a realist evaluation. Therefore, identifying the exact $\mathrm{CMO}$ configuration is an important part of the analysis. In the present study, the researchers determined the $\mathrm{CMO}$ configuration by first identifying the service outcome, which they then used as a guide to iteratively review the data material until the causal mechanisms underlying the outcome were understood. The analysis was directed by theorising about 'how this outcome came about' (mechanism) and 'what about the TADA service generated this outcome' (context). Generating this $\mathrm{CMO}$ configuration was as retroductive as it was iterative, i.e. the $\mathrm{CMO}$ configuration revealed the interaction and generative association between the context and the mechanism, in turn leading to the outcome, which in turn ultimately illuminated the essential connections between the individual components of the $\mathrm{CMO}$ configuration.

A central tenet of realist evaluations is the understanding of the architecture of the phenomenon - in the current study, this meant comprehending how stakeholders designed the service, structurally speaking, to deliver the intended service outcomes. By conceptualising the architecture of the TADA service, its service pathways and delivery roles were also revealed. This architecture is presented below, in the Results section.

The quotes displayed below were translated by the first author from Norwegian into English and then back-translated from English to Norwegian by an independent party. The third author is an active TADA practitioner who is responsible for developing and delivering the CBT training curriculum used by the service. Thus, the programme theories and architecture were member-checked by the third author as they emerged. The qualitative software programme NVivo was used to manage the data $[31,48,49]$. To ensure the informants' anonymity, the quotes presented in the Results section were assigned arbitrary numbers from 1-12. That said, each quote is identified according to the profession of the speaker, i.e. whether the anonymised service practitioner specialised in psychology or dentistry.

\section{Results}

In answering the question of what works, for whom, under which circumstances, and how and why, our analyses identified four $\mathrm{CMO}$ configurations involving contextual factors at the macro and meso levels, each of which affected the service outcome. Not all the data are presented here; instead, we included only those findings depicting how the stakeholders theorised the structural features of the TADA service in terms of what works, for whom, under which circumstances, and how and why. Micro-level data will be presented elsewhere (paper in review).

The architecture of the TADA service and the various service pathways produced for the service to achieve its end goal - namely, TADA patients with restored oral health and relief from dental anxiety are presented in Fig. 2. Additionally, Fig. 2 shows how the TADA service pursues an interdisciplinary 
approach by combining psychology and dentistry into a single service. Operationally, this means that the service is divided into two departments, each of which consists of a separate team tasked with delivering a specific aspect of the service. The first department (the green section in Fig. 2) is staffed by an interdisciplinary team whose focus is on the psychological dimensions of the TADA service. Specifically, this team is charged with mitigating or alleviating trauma or anxiety among patients. The second department (the yellow section in Fig. 2) is focused on oral health, with their primary objective being the restoration and maintenance of the patients' oral health.

As mentioned above, the TADA team comprises practitioners from psychology and dentistry. The psychological department of the TADA team is tasked with mitigating or alleviating trauma or anxiety among TADA patients via CBT sessions, the purpose of which is to arrive at a fuller understanding of the interconnections between cognition, behaviour and emotion, after which a collaborative, active and direct approach can be taken to challenging and resolving associated dysfunctions [41]. A major feature of CBT involves exposing the patient to the causes or triggers of dysfunction (i.e. in-vivo exposure therapy), which, in the current study, was the dental setting. In the TADA service, dental practitioners execute the exposure therapy, which is intended to gradually but directly desensitise patients to the anxiety-provoking aspects of the dental experience [18]. Although the treatment duration is typically 12 sessions, field experience has shown that, often, additional treatment sessions are needed, with the number of sessions correlating with the severity of the trauma experienced by the patient. Any dental treatments administered by the psychologically oriented TADA team during exposure therapy are aimed only at alleviating anxiety, not restoring oral health. The purpose here is to motivate patients to continue to pursue and eventually achieve dental restoration via the dental follow-up team after their anxiety has been effectively managed.

Upon completion of the CBT therapy, or in cases in which patients are assessed as non-responsive to therapy, the dental follow-up team is tasked with restoring the patients' oral health. The follow-up team's primary responsibility in this regard is to restore patients' oral health to an acceptable standard: no pain, no discomfort, no severe ailments, e.g. cavities, satisfactory functionality, and the capacity to communicate and participate in social settings without teeth pain or other dental complications [42].

We developed our programme theories based on causal chains between contexts, mechanisms and outcomes in the TADA service architecture, as presented in Table 3 below, resulting in several discrete CMOCs. 
CMOCs: The Building Blocks for Programme Theories

\begin{tabular}{|c|c|c|c|}
\hline CMO & Context + & Mechanism = & Outcome \\
\hline \multirow[t]{2}{*}{$\begin{array}{l}\text { Number 1, } \\
\text { relates to } \\
\text { Programme } \\
\text { Theory } 1\end{array}$} & $\begin{array}{l}\text { State-funded service. Deliver both anxiety } \\
\text { treatment and dental restoration. The cost of } \\
\text { dental restoration needs, for many of these } \\
\text { patients, is unaffordable. The annual (for } 2020 \text { ) } \\
\text { budget for the service was } 85 \text { million Norwegian } \\
\text { kroners (around } 8 \text { million euros). }\end{array}$ & $\begin{array}{l}\text { Increased } \\
\text { accessibility. } \\
\text { a vulnerable } \\
\text { group } \\
\text { accesses } \\
\text { services they } \\
\text { would } \\
\text { otherwise be } \\
\text { unable to } \\
\text { afford. This } \\
\text { makes it } \\
\text { easier for the } \\
\text { patient to } \\
\text { improve their } \\
\text { oral health. }\end{array}$ & $\begin{array}{l}\text { The } \\
\text { immediate } \\
\text { outcome for } \\
\text { patients: } \\
\text { accessibility } \\
\text { increases } \\
\text { service } \\
\text { uptake. Ripple } \\
\text { effects for } \\
\text { patients: } \\
\text { improved } \\
\text { quality of life }\end{array}$ \\
\hline & & $\begin{array}{l}\text { Altered focus: } \\
\text { The service } \\
\text { shifts from } \\
\text { private to } \\
\text { public. }\end{array}$ & \\
\hline $\begin{array}{l}\text { Number 2, } \\
\text { relates to } \\
\text { Programme } \\
\text { Theory } 2\end{array}$ & $\begin{array}{l}\text { National guidelines set by the Norwegian } \\
\text { government are open to interpretation. Some } \\
\text { patients are in difficult life situations and may not } \\
\text { always benefit from the full CBT dimension of the } \\
\text { TADA service. Patients are heterogeneous in } \\
\text { character. }\end{array}$ & $\begin{array}{l}\text { The TADA } \\
\text { teams tailor } \\
\text { their approach } \\
\text { by learning } \\
\text { what the } \\
\text { patient needs } \\
\text { and searching } \\
\text { local } \\
\text { resources in } \\
\text { order to meet } \\
\text { these needs. }\end{array}$ & $\begin{array}{l}\text { The service } \\
\text { delivers } \\
\text { treatment, } \\
\text { improving the } \\
\text { oral health of } \\
\text { patients who } \\
\text { follow the } \\
\text { service } \\
\text { pathway. Not } \\
\text { all patients } \\
\text { are relieved of } \\
\text { dental anxiety. }\end{array}$ \\
\hline $\begin{array}{l}\text { Number 3, } \\
\text { relates to } \\
\text { Programme } \\
\text { Theory } 3\end{array}$ & $\begin{array}{l}\text { The Directorate of Health controls the service. } \\
\text { There is a lack of common meeting arenas. There } \\
\text { is a lack of explicit leadership and guidelines from } \\
\text { the Directorate of Health. Poor communication } \\
\text { across teams (nationally and regionally). County } \\
\text { legislations affect service delivery. }\end{array}$ & $\begin{array}{l}\text { TADA teams } \\
\text { become self- } \\
\text { reliant and } \\
\text { protectionist } \\
\text { in their work. }\end{array}$ & $\begin{array}{l}\text { TADA teams } \\
\text { work } \\
\text { cohesively as } \\
\text { a team, but } \\
\text { separately. }\end{array}$ \\
\hline
\end{tabular}




\begin{tabular}{|c|c|c|c|}
\hline CMO & Context + & Mechanism = & Outcome \\
\hline $\begin{array}{l}\text { Number 4, } \\
\text { relates to } \\
\text { Programme } \\
\text { Theory } 4\end{array}$ & $\begin{array}{l}\text { Increased incidence and severity of torture } \\
\text { methods in countries from which migrants have } \\
\text { fled. Accounts from the Directorate of Health } \\
\text { reveal that few torture survivors have applied for } \\
\text { this service. Teams have adjusted to } \\
\text { accommodate patients faster. There is possibly a } \\
\text { lack of sufficient advertisements for this service. } \\
\text { When fleeing from conflict areas and trying to } \\
\text { resettle in a new country, dental anxiety and/or } \\
\text { dental restoration are not necessarily prioritised. } \\
\text { This patient group undergoes a long asylum } \\
\text { interview in which their backgrounds are checked } \\
\text { and they are asked to describe their torture } \\
\text { experiences. Being asked to do so again may, } \\
\text { therefore, be exhausting. }\end{array}$ & $\begin{array}{l}\text { The patients } \\
\text { may } \\
\text { experience the } \\
\text { service as too } \\
\text { overwhelming. } \\
\text { Their dental } \\
\text { health is not } \\
\text { prioritised at } \\
\text { the given time. } \\
\text { They are } \\
\text { unaware such } \\
\text { a service } \\
\text { exists. }\end{array}$ & $\begin{array}{l}\text { The service is } \\
\text { unable to } \\
\text { reach and } \\
\text { accommodate } \\
\text { patients who } \\
\text { suffered from } \\
\text { torture. }\end{array}$ \\
\hline
\end{tabular}

Programme Theory 1: Subsidising the TADA service means oral healthbecomesa public project and dental avoidance behaviours become a public health concern. This consequently improves patient access and service uptake

In Norway, patients over 20 years old are responsible for paying for their own dental care - the TADA service, however, is an exception. This is because both relevant data sources and service developers have indicated that the cost of dental treatment for TADA patients is prohibitive. Without assistance, these patients would be incapable of affording the restoration of their oral health. The TADA service is therefore subsidised by the state, providing dental care free of charge for this population. This provision covers the cost of both dental rehabilitation and anxiety treatment, allowing the TADA service to address and ameliorate the major precursor to deteriorated oral health among TADA patients: anxiety and trauma. Via state subsidies and a bi-dimensional approach, the TADA service makes dental treatment more accessible and oral health more attainable for this population, addressing not just cost issues but anxiety-related behavioural issues as well. Consequently, the TADA service is contributing to a cultural shift in which dental health care is increasingly being viewed as a public rather than private provision, foregrounding oral health as a public health concern. As depicted in the CMOC (see Table 3), these trends have had the immediate outcome of increasing dental service uptake, with a total of 1186 enrolled patients in 2018. Additionally, the service developers believe such enrolment is having ripple effects for these patients, whom they describe as feeling more capable of reengaging in society and experiencing improved quality of life.

It is clear that such a service like this, it's costly, yeah ... but it provides quality of life... it has large effects, then, for a lot of people... (Interview 3 , service developer within dentistry)

I think that when I see a bill of 200,000 [Norwegian kroner, equivalent to 20,000 euros], I think, 'money well spent.' For there may be someone who gets back to work, one who manages to be a mother or father again and can live normally, as a normal human being - get their dignity back. So, I'm pretty sure that Norway will get the TADA money back in, yeah ... with good returns later, not that year but in a few years. I'm pretty sure it will earn itself back. (Interview 10, service developer within psychology) 
... I think that the TADA service has succeeded in helping many people. Oh lord, compared to when I started ... a lot of people get help. It's been on the agenda; everyone knows about it. This is not a thing the dentists can push away anymore, sweep away. So, the project is successful, I think. (Interview 8, service developer within dentistry)

Programme Theory 2: Catering to a heterogeneous patient group means adapting and tailoring the service to regional resources and patient requirements

The service policy of the Norwegian Health Directorate is loosely prescriptive. This allows a degree of regional autonomy and professional interpretation regarding how practitioners should deliver the service. The patient group is described as heterogeneous (suffering from various traumas resulting from torture or abuse, or from dental phobia) and complicated in terms of socioeconomic status, health, and other life circumstances. Service developers, therefore, have viewed a standardised service as inadequate and have instead adapted the service in light of local resources and patient requirements. Local resources include the use of anaesthesia and the incorporation of additional psychological therapy, but may also entail pausing the patient during the service pathway. Pausing the patient means postponing therapy or dental restoration until the patient considers themselves ready to proceed. By adapting the service to local resources, the TADA practitioners can tailor the service according to what it can realistically offer and to what the patient can realistically achieve. This, however, implies that some patients may receive full dental restoration without finishing their anxiety treatment and may consequently not overcome their fear of the dental setting (including examinations). Nonetheless, by being flexible and tailoring services according to available local resources and realistic goals, the TADA practitioners can improve the oral health of their patients. In their own words, the practitioners believe in finding the service pathway best suited to the needs and capacities of each patient.

They are difficult to catch because they often have comorbidities; they have other issues in their life. And we have patients that I have given anaesthetics to [for dental restoration] and put on hold, because - I remember clearly a young lady, like 27-28 years, child welfare services were at the door, new little girl, little daughter, beaten by the partner, had a security alarm and eh ... lived what you would call a difficult upbringing. Of course, she was being assessed for PTSD, eh ... and then she started feeling pain in her teeth, so she felt she needed to deal with that, and for her to come once a week for CBT and twice a week for PTSD treatment and meet with child welfare services and no ... [...] lt became too much for her. (Interview 3, service developer within dentistry)

What is being successful for the patients? [...] we have limited the goal. Previously, the goal was to go to the dentist regularly, but now we have seen that it's too much for her in this round, so then we have said that the goal for this round is to have a clinical examination, so that she can receive treatment under anaesthesia if necessary. [...] but sometimes we just have to, we just have to adjust the goals a bit. If people are traumatised and are not capable of carrying out the treatment with tartar and sprayer and drill and all of those. (Interview 4, service developer within psychology) 
Programme Theory 3: A national service, operated by individual satellites, leads to a lack of communication, nationally and regionally, and isolation

The Norwegian Directorate of Health seeks to maintain a similar standard of service across the country. This is achieved nationally by controlling service guidelines, implementing regulations and providing policies. However, the management of local resources and logistics is the responsibility of counties and TADA teams. This county-level compartmentalization creates disjunctures between regional teams and between the intentions of teams and those of the Directorate of Health, hampering the delivery of the same standard of service across the country. These disconnects, coupled with the lack of bilateral meeting arenas (in which TADA teams can meet), the absence of explicit leadership on how to interpret ambiguities in the implementation of the service, and gaps in ministry guidelines, fosters team isolation and impedes effective communication and collaboration.

As a result, individual TADA teams have become more self-reliant and efficient at resolving challenges with local solutions, which are often not shared with other teams. Despite these limitations, and perhaps because of them, each TADA team has become more cohesive.

I probably feel that we, to a high degree, have become satellites at each of our clinics who've developed our own way of working ... And, maybe a bit too protectionist in that, and instead of using the days - the semi-annual meetings for something very constructive, it's very quick for everyone to just sit down to tell how they are doing it. And, we develop some solutions for things, because, the guidelines are okay - but less specific [...] And when there is no common location there are no common meetings between the psychologists, for example, there is no regular hospitality with each other, no common meeting points ... No line between us and management. (Interview 11, service developer within psychology)

The Directorate of Health wanted this particular service to be a public offer. In that its public, they would also be in control of it [...] in that it would be an equal offer... and that people working with training ... according to a plan, and that the plan was quality assured ... (Interview 7, service developer within dentistry)

Internally, we are stuck, each in our own cave. (Interview 4, service developer within psychology)

Programme Theory 4: Lack of recruitment to the TADA service is explained by challenges patients experience because of the migration process and poor dissemination practices

There has been a large increase in migrants and asylum seekers from countries in which torture occurs, so much so that the TADA service is currently struggling to accommodate the dental restoration and dental anxiety treatment needs of all of these patients. Although patient demographics from the TADA service indicate that a little under 2 per cent (21/1186 patients in 2018) are torture survivors, Norway is believed to host a far greater number: between 10,000 and 35,000. The TADA teams have thus been experimenting with a design intended to increase service access by this population by minimising the waiting time for admission and ensuring the presence of independent interpreters - yet, thus far, this 
approach has been minimally effective. One explanation for this, according to stakeholders, is that torture survivors are already overwhelmed by the extensive paperwork they must complete when entering Norway, making registering for the TADA service an additional burden. Another potential explanation is that the oral health of such patients is simply not a priority when compared to the challenges involved in resettling in a new country and attempting to fit in with a new society.

Service developers have considered a third possibility: patients may be unaware of the TADA service due to a lack of flyers, posters and other advertisements at asylum facilities or transcultural centres, and that 'word of mouth' is just not effective at reaching this population. Simply put, the TADA service is currently unable to treat torture survivors, one of its three target groups, as these patients are either already overburdened or are not even aware that the service exists.

A lot of them feel the TADA treatment is too extensive. They have to get to a certain place, so they would rather have treatment at a clinic that is near the school where they attend ... where they are often attending introductory programmes ... to be gone for a whole day, well, that can be difficult.[...] I definitely think they should have an offer, but I'm not really sure if ... like, a lot of people have told me, 'No, do I need to speak to a new psychologist? I have a psychologist. Can't he/she do this?'(Interview 1, service developer within dentistry)

[the informant imitates a patient],'You know what, now I have finally got, eh, after many years fleeing, then I finally got a residence permit, got myself a house, Norwegian of course' - There is a lot to establish in Norway, to start such an anxiety treatment or dental treatment is not on the priority list, so we have tried very hard to recruit them, despite persistent attempts to make a deal with the municipality about a twoweek waiting list, and we have invited them to cooperation meetings. (Interview 4, service developer within psychology)

\section{Discussion}

Realist evaluations are increasingly popular within health research, as they have the potential to better understand the vital contexts that trigger working mechanisms and that result in enhanced service outcomes [33]. Our realist evaluation of the TADA service, a dental anxiety service catering to patients who have survived torture or abuse or who suffer from dental phobia, revealed that service developers are guided by four main programme theories, each of which is useful for describing the generation of service outcomes at a structural level. The contextual elements, working mechanisms and resulting outcomes, together comprising the building blocks of our programme theories, are discussed below in parallel to other studies in order to highlight the implications of the current study and make some valuable recommendations. By studying the contexts, mechanisms and outcomes of the TADA service, our findings can assist in general programme development and can aid policy makers seeking to implement a similar service in a local setting or working to improve existing programmes. Our research can also contribute to existing knowledge on dental anxiety treatment and services for vulnerable patient groups. 
Our first programme theory describes how, in a context in which a service is state-subsidised, oral health has shifted from being a private matter to becoming a public concern, and has, for TADA patients, increased service uptake. Seen from a service perspective, this means that by removing financial barriers, services such as the TADA service can reach patient populations that would otherwise avoid general dental services. The determination about whether to subsidise dental services is an ongoing issue in Norway. As it currently stands, dental examinations and restorations are considered the private responsibility of adults. Therefore, by providing a bi-dimensional service, one which covers psychological needs (through CBT) and dental needs, free of charge for a target patient population, the TADA service deviates from the Norwegian norm.

If we consider what subsidising the TADA service means for the Norwegian welfare state, it becomes clear that the state is taking the neglect of oral health seriously enough to establish equitable oral health policies at a societal level $[50,51]$. Including oral health services as a government priority represents an essential step not just in tackling oral diseases but also in reconceiving oral health as a critical aspect of a more holistic health picture $[52,53]$. Oral health inequality is currently the subject of heated debate because of the increasingly wide societal gap between those who are able to afford dental care and those who are not [50]. Consequently, when access to dental care is unequal, social inequality worsens, as only those able to afford such care benefit [50,54-57].

Looking deeper into the ramifications of state-subsidised dental service, one study examined Norwegians patients' willingness to pay for dental care after receiving CBT, practising relaxation methods or being sedated in the dental restoration process [58]. The results of this study revealed that patients were less willing to pay for such procedures pre-treatment ( 24 per cent, $\mathrm{N}=65$ ) but were more willingly to do so post-treatment (71 per cent). Based on these findings, the authors argued that patients might avoid treatment unless or until it is subsidised [58]. Looking beyond Norway, in an international context, Gulliford and colleagues [59] discussed the meaning of health care access within the National Health Service (NHS) in Britain and found financial barriers to be a significant deterrent to service utilisation and access for vulnerable patients $[59,60]$.

Our first programme theory also includes the notion of ripple effects, revealing an extension of the service outcome. As Programme Theory 1 describes, by providing a service through which patients can have their psychological needs met and their oral health restored, the service deliverers are discovering that patients are reengaging in society and experiencing an improved quality of life. With restored oral health, these patients' fear of 'revealing' their mouth $[4,6]$ is diminished, and they are moreover able to participate in society with more functional oral health, thereby allowing them to communicate, eat, swallow, smile and taste without discomfort [61, 62].

Research from countries such as Germany, Sweden and Brazil have assessed the link between dental fear, dental pain, dental access and quality of life through quantitative measures that have given a clear indication of the impact these factors have on one's quality of life [63-65]. 
As a conclusion to our first programme theory, which assessed the effect of successful service uptake on the quality of life of TADA patients, we recommend that Norwegian policy makers continue to subsidise the TADA dental service. For international policy makers looking to implement such a service, we emphasise how important state subsidies are to ensuring the success of the service.

Our second programme theory specifically addressed how the TADA service works, for whom, under which circumstances, with what resources, and why. Our interviews revealed that service developers acknowledge that some methods will work for some patients, but not others, and that this depends in large part on the local resources a team can manage to acquire as well as what patients can realistically achieve. The context in Programme Theory 2 explicates two essential elements that must be considered in the structure of the service. First, the TADA patient group is heterogeneous, with many patients experiencing difficult life situations that make it challenging for them to attend or complete the anxiety treatment provided by the TADA service. Second, as national guidelines on the service are loosely prescriptive, professional practice and discretion are essentially the responsibility of individual teams at the county level. As our illustration of service pathways reveals (see Fig. 2), the TADA service prioritises the alleviation of anxiety, and thus CBT treatment is the first-line treatment. However, should a TADA team discover that a patient is not responding to this treatment, the current structure of the TADA service permits the team to autonomously investigate what local resources are available to continue to accommodate the patient's denta/ needs. By acquiring additional resources to address patients' dental needs, the TADA teams can ensure that the service still meets its end goal of providing patients with acceptable oral health (see Fig. 2). Nevertheless, in such cases, should the patient continue to suffer from untreated anxiety, the dental restoration needed to achieve acceptable oral health will often require anaesthetics or other sedative methods. From a service perspective, this would mean that even though the TADA service has not necessarily met all of the patient's psychological needs, it has successfully restructured its bi-dimensional service outcome by tailoring the service to accommodate the patient's dental needs.

Tailoring and restructuring service outcomes are beneficial for the patient insofar as doing so matches what the patient can realistically achieve. Nevertheless, these measures have mixed implications for practice. The first implication is related to cost, as in cases where dental restoration under anaesthetics is needed, the cost of such a procedure is increased, with the state being required to cover the cost via subsidies. Second, patients who receive a full dental restoration without successful treatment of their anxiety may continue to practise avoidance behaviours. Third, the specifics of the TADA service vary across Norway because of corresponding variations in local resources. How and when local resources are allocated will depend on factors such as the accessibility of anaesthetics within a county, how well individual TADA teams manage their time and waiting lists when pausing patients, and to which dental follow-up team they are able to refer their patients.

Balancing between customising the service to meet individual patient needs and adhering to organisational standards, which is typical within health and welfare services, can be overwhelming for TADA teams. Through the lens of Lipsky's theory of street-level bureaucracy [63], we can view service 
deliverers as street-level bureaucrats via their position as front-line workers and facilitators of the TADA service. In their position, they are required to interpret organisational guidelines and practise professional discretion through their direct contacts with patients. For their part, the TADA teams have a sense of autonomy, as the TADA guidelines do not explicitly mandate how the service should meet its end goal of providing patients with an acceptable level of oral health (see Fig. 2). Furthermore, as depicted in Fig. 2, TADA teams have implemented a variety of methods for reaching the same goal; thus, their position as street-level bureaucrats gives them room to adapt the TADA service with a bottom-up approach [66]. Nevertheless, conflicts could arise if the service were to shift to a top-down approach. This could occur if, for example, the Directorate of Health were to expect the service deliverers to meet the criterion of treating all aspects of patients' anxiety and dental needs. Top-down approaches are often concerned with controlling a service, thereby minimising discretion while attaining higher-level goals [67]. This would require a new structure and likely lead to conflict with the service deliverers, as they typically do not manage to realise all of the benefits of the CBT dimension offered by the service. In such cases, the service deliverers would be prone to fail in the street-level bureaucracy, as their relevance would be diminished [68]. Nevertheless, as the service is currently structured, the service deliverers can advantageously practise discretion, allowing them to customise the service to accommodate the patients' needs while avoiding being overwhelmed by the Directorate of Health's top-down expectations. The TADA service's approach in this respect reflects a hybrid solution incorporating top-down and bottom-up approaches $[66,69,70]$.

Our recommendations for policy makers are, therefore, to continue practising this hybrid bottom-up and top-down approach, permitting teams to interpret the national guidelines to match their local context. With a hybrid approach, street-level bureaucrats become service enablers rather than service constraints $[66,68-70]$.

Programme Theory 3 continues the reflection on the role of service deliverers by describing the contextual landscape of the TADA service, including the lack of joint meeting arenas and centralised leadership, and the ambiguity generated by county-level compartmentalization and poor communication between teams. In such a context, our analyses revealed that teams are compelled to be more self-reliant and protectionist about their work and, as an outcome, they must operate in isolation. Therefore, the structure of the service, as it presently stands, is not conducive to the cultivation of a national service.

Nevertheless, working in isolation was also found to enhance the sense of team membership and thus foster greater cohesion. This is because when teams share a set of assumptions and demonstrate likeminded thinking, cohesion is strengthened [71]. This is important, as stronger team cohesion translates to better performance, primarily because the team members can trust each other's professional skills and lean on each other's discretion, e.g. the psychologist's judgement and the dental practitioner's judgement.

Notwithstanding, as the contextual backdrop of the TADA service is devoid of national meeting arenas and bilateral meeting places, team-based solutions to service challenges typically remain within each 
team. This in turn prevents teams from learning from each other's experiences, which is problematic given that local solutions can be beneficial not just for other teams but for the TADA service as a whole. Without such inter-team communication, each team remains 'stuck, in our own cave'(Interview 4). Especially now, as the COVID-19 pandemic rampages across localities, how can teams adapt without broader cooperation? Would not cross-fertilisation of local solutions be beneficial? With this in mind, we recommend, for both local and international policy makers, that collective venues and platforms be installed during programme implementation to inspire innovation and cultivate a shared culture across teams. Such venues could range from annual service conferences that encourage the exchange of local solutions to interactive digital platforms on which cases could be easily shared and discussed - a timely option given the current pandemic. We assume that by introducing resources such as shared meeting arenas and bilateral meeting places (be they virtual or face to face), TADA teams could greatly increase their knowledge and expand their awareness via the exchange of professional experiences, contextualised at the local level, concerning what works for which types of patients.

The research presented in Programme Theory 4 specifically addresses the survivors of torture. In this regard, when assessing the TADA service as a whole, it can be considered a success in terms of its uptake and extended waiting list of patients in need of the service. However, when disaggregated into subcategories, we found that the service is unable to accommodate torture survivors. As Programme Theory 4 shows, Norway is home to between 10,000 and 35,000 torture survivors [15]. However, according to documents retrieved from the Norwegian Directorate of Health, as of 2018, only 21 torture survivors (representing less than 2 per cent of the TADA patients) were admitted to the TADA service. The remaining 98 per cent of TADA patients are either abuse survivors or sufferers of dental phobia. Therefore, the current design of the TADA service appears to be ineffective at reaching and enrolling torture survivors. Stakeholder interviews conducted for Programme Theory 4 described three different contexts that may account for this deficiency. First, there appears to be a lack of contextual understanding before service implementation, which could in turn explain the failure of the TADA service to reach this target population [72]. Additionally, the literature on torture survivors is limited and focuses mostly on the quantitative reporting of their dental status, which is often poor [24, 28], thereby underscoring the need for the TADA service. One Australian study [73] found that barriers such as long waiting lists and interpreter services discourage survivors of torture from enrolling in available dental services. Nonetheless, as the contextual data for Programme Theory 4 demonstrate, the TADA teams adjust waiting times and invite independent interpreters to participate in the service. That said, Programme Theory 4 also found that the requirements for asylum seekers are overly bureaucratic and often overwhelming for patients. At the same time, if the torture survivors are already receiving psychological assistance elsewhere, why would they benefit from - or even seek out - additional psychological help from the TADA service? It is possible that the TADA service's bi-dimensional approach may substantively affect the uptake of torture survivors. We therefore recommend that the service scrutinises the specific needs of torture survivors to assess whether they require both psychological and dental treatment. Building on this premise, future research should collect more data from torture survivors 
and examine the different contexts discussed in this programme theory to determine why the observed outcome is occurring.

Study limitations

This paper presented an in-depth study, from the perspective of TADA service developers, of some of the theories underlying the structure and functions of the service. A number of insights were generated, but not without some limitations. For instance, it would be advisable to include additional consultations and perform focus-group interviews with stakeholders to refine the programme theories - a standard practice within realist evaluations $[33,47]$. That this was not done in the current study was a pragmatic choice, one that reflected time constraints, the multiplicity of roles and the distance between informants.

Another limitation of this study was the small sample size of stakeholders: 12 service developers who, at the time the interviews were conducted, were also playing an active role in service delivery and were representative of discrete regional service settings. Although sample sizes are typically smaller for realist evaluations, as with most qualitative studies, the reliability of our findings could have been improved with a larger number of informants. Accordingly, we recommend the performance of case-specific studies with larger samples to test the programme theories presented in the present work in order to enhance their reliability.

\section{Conclusion}

The bi-dimensional structure of the TADA service, which provides both anxiety and dental treatment free of charge, has increased service uptake among a vulnerable patient population that would otherwise avoid such services. Our findings indicate that this service uptake is on the whole beneficial, as service deliverers have received positive feedback from patients, who have asserted that the service has helped them reengage with society and has improved their quality of life. Additionally, with an enrolment of 1186 patients, the service can be considered successful. However, when disaggregated into the three subcategories comprising the target population - torture survivors, abuse survivors and patients suffering from dental phobia - it becomes clear that patients with a past history of torture are vastly underrepresented (only 2 per cent of the serviced population). To explain this, we implicated several possible contextual factors, yet more research on this patient population is certainly warranted. Generally speaking, however, for patients who make use of the TADA service, its hybrid bottom-up and top-down approach has proven beneficial; likewise for the service teams, for whom the hybrid approach has permitted the opportunity to practise professional discretion while at the same time tailoring the service to meet the needs of individual patients. This capacity has proven to be especially valuable in cases where the service outcomes of patients must be adapted due to complex, often difficult life situations and non-responsiveness to CBT in order to ensure the accommodation of their dental needs and the maintenance of their overall oral health.

\section{Abbreviations}


TADA

torture, abuse and dental phobia

C

context

$\mathrm{M}$

mechanisms

0

outcome

$\mathrm{CMOC}$

context + mechanism $=$ outcome configuration

\section{Declarations}

\section{Ethics approval and consent to participate}

Voluntary participation was based on informed consent. Written consent was obtained from study participants. The Norwegian National Centre of Research Data approved this research (Project No: 619754).

Consent for publication

Informed consent to study participation included consent for publication.

Availability of data and materials

Data material and analyses are conducted in Norwegian. Upon reasonable request, data material will be translated and made available from the corresponding author.

Competing interests

The authors declare that they have no competing interests.

\section{Funding}

The Oral Health Centre of Expertise, Rogaland, Norway, is an independent public research institution performing practice-oriented research. The Oral Health Centre of Expertise, Rogaland, Norway funds the $\mathrm{PhD}$ project of the lead other, and this study pertains the lead other's PhD. Their role in funding did not impact the study design, analyses, interpretation of data or writing the manuscript.

Additional information

Correspondence to Emilie Bryne

Affiliations 
Oral Health Centre of Expertise, Rogaland, Norway

Emilie Bryne, Kjersti Berge Evensen, Vibeke Hervik Bull

Faculty of Social Sciences, University of Stavanger, Norway

Emilie Bryne, Sarah Patricia Catherine Duff Hean

Authors' contributions

$\mathrm{EB}, \mathrm{SH}, \mathrm{KB}, \mathrm{VB}$ were involved in formulating the problem, structuring the study's design, and interpreting the results. EB wrote the main part of the manuscript. All authors accept responsibility of authorship and agree to be accountable for this article.

\section{Acknowledgements}

We are grateful for the employers, nationally, at the TADA services, the Regional Oral Health Centres of Expertise, and the Directorate of Health for helping the conduct of this study.

\section{References}

1. Locker D, Clarke M, Payne B: Self-perceived Oral Health Status, Psychological Well-being, and Life Satisfaction in an Older Adult Population. Journal of Dental Research 2000, 79(4):970-975.

2. Slade GD: Measuring oral health and quality of life: Department of Dental Ecology, School of Dentistry, University of North Carolina; 1997.

3. Ng SK, Leung WK: Oral health-related quality of life and periodontal status. Community dentistry and oral epidemiology 2006, 34(2):114-122.

4. Lockhart PB, Brennan MT, Thornhill M, Michalowicz BS, Noll J, Bahrani-Mougeot FK, Sasser HC: Poor oral hygiene as a risk factor for infective endocarditis-related bacteremia. The Journal of the American Dental Association 2009, 140(10):1238-1244.

5. Petersen PE, Kwan S: Equity, social determinants and public health programmes - the case of oral health. Community Dentistry and Oral Epidemiology 2011, 39(6):481-487.

6. Locker D: Psychosocial consequences of dental fear and anxiety. Community Dentistry and Oral Epidemiology 2003, 31(2):144-151.

7. The World Health Organization: The global burden of oral diseases and risks to oral health. Bull World Health Organ 2005, 83(9):661-669.

8. Norwegian Directorate of Health: Tilrettelagte tannhelsetilbud for mennesker som er blitt utsatt for tortur, overgrep eller har odontofobi (Facilitated dental health services for people who have been subjected to torture, abuse or odontophobia). In. Oslo: Helsedirektoratet (The Health Directorate); 2010. 
9. Ministry of Health and Care Services: Report to the Storting (white paper) Tilgjengelighet, kompetanse og sosial utjevning (Accessibility, expertise and social leveling). In: Tilgjengelighet, kompetanse og sosial utjevning -Framtidas tannhelsetjenester (Accessibility, expertise and social equalization -the futures dental health services). 2006-2007.

10. Öst L-G, Skaret E: Cognitive behavioral therapy for dental phobia and anxiety: John Wiley \& Sons; 2013.

11. Gordon D, Heimberg RG, Tellez M, Ismail Al: A critical review of approaches to the treatment of dental anxiety in adults. Journal of Anxiety Disorders 2013, 27(4):365-378.

12. Wide Boman U, Carlsson V, Westin M, Hakeberg M: Psychological treatment of dental anxiety among adults: a systematic review. European Journal of Oral Sciences 2013, 121(3pt2).

13. Åstrøm AN, Ekback G, Klock K, Unell L: En femårsstudie av tannhelse og tannhelsevaner blant 65åringer i Hordaland, Sogn og Fjordane og Nordland. Tidende, The Norwegian Dental Journal 2007:434-440.

14. Vujicic M, Buchmueller T, Klein R: Dental Care Presents The Highest Level Of Financial Barriers, Compared To Other Types Of Health Care Services. Health Aff (Millwood) 2016, 35(12):2176-2182.

15. The Norwegian Red Cross: Torturert og glemt? Identifsering og rehabilitering av torturutsatte i Norge (Tortured and forgotten? Identification and rehabilitation of torture victims in Norway). In. Oslo: Røde Kors (The Red Cross); 2020.

16. Hafstad GS, Augusti E-M: Ungdoms erfaringer med vold og overgrep i oppveksten. En nasjonal undersøkelse av ungdom i alderen 2019, 12.

17. Thoresen S, Hjemdal O: Vold og voldtekt i Norge: En nasjonal forekomststudie av vold $i$ et livsløpsperspektiv (Violence and Rape in Norway: A National Prevalence Study of Violence in a Lifespan Perspective). In., vol. Vol. 1/2014 Oslo: Nasjonalt kunnskapssenter om vold og traumatisk stress (The Norwegian Centre for Violence and Traumatic Stress Studies) Nasjonalt kunnskapssenter om vold og traumatisk stress Oslo; 2014.

18. American Psychiatric Association: Diagnostic and statistical manual of mental dissorders, 5th edn. Arlington, VA: American Psychiatric Publishing; 2013.

19. Strøm K, Skaare AB, Willumsen T: Dental anxiety in 18-year-old Norwegians in 1996 and 2016. Acta Odontologica Scandinavica 2019, 78(1):13-19.

20. Sillevis Smitt H, de Leeuw J, de Vries T: Association Between Severe Dental Caries and Child Abuse and Neglect. Journal of Oral and Maxillofacial Surgery 2017, 75(11):2304-2306.

21. Stalker CA, Russell BDC, Teram ELI, Schachter CL: Providing dental care to survivors of childhood sexual abuse: Treatment considerations for the practitioner. The Journal of the American Dental Association 2005, 136(9):1277-1281.

22. Walker EA, Milgrom PM, Weinstein $P$, Getz T, Richardson R: Assessing abuse and neglect and dental fear in women. Journal of the American Dental Association 1996, 127(4):485-490.

23. Singh $\mathrm{H}$, Bhaskar D, Rehman R: Psychological aspects of odontophobia. International Journal of International Medical Research 2015, 1(6):210-212. 
24. Singh HK, Scott TE, Henshaw MM, Cote SE, Grodin MA, Piwowarczyk LA: Oral health status of refugee torture survivors seeking care in the United States. American journal of public health 2008, 98(12):2181-2182.

25. Wilson FA, Wang Y, Stimpson JP, McFarland KK, Singh KP: Use of dental services by immigration status in the United States. J Am Dent Assoc 2016, 147(3):162-169 e164.

26. Willumsen T: Dental fear in sexually abused women. European Journal of Oral Sciences 2001, 109(5):291-296.

27. Willumsen $\mathrm{T}$ : The impact of childhood sexual abuse on dental fear. Community Dent Oral Epidemiol 2004, 32(1):73-79.

28. Høyvik AC, Lie B, Willumsen T: Dental anxiety in relation to torture experiences and symptoms of post-traumatic stress disorder. European Journal of Oral Sciences 2018, 0(0).

29. Ehring T, Welboren R, Morina N, Wicherts JM, Freitag J, Emmelkamp PMG: Meta-analysis of psychological treatments for posttraumatic stress disorder in adult survivors of childhood abuse. Clinical Psychology Review 2014, 34(8):645-657.

30. Patel N, C de C Williams A, Kellezi B: Reviewing outcomes of psychological interventions with torture survivors: Conceptual, methodological and ethical Issues. Torture 2016, 26(1):2-16.

31. Pawson R, Tilley N: Realistic evaluation: sage; 1997.

32. Shearn K, Allmark P, Piercy H, Hirst J: Building realist program theory for large complex and messy interventions. International Journal of Qualitative Methods 2017, 16(1):1609406917741796.

33. Wong G, Westhorp G, Manzano A, Greenhalgh J, Jagosh J, Greenhalgh T: RAMESES II reporting standards for realist evaluations. BMC medicine 2016, 14(1):96.

34. Emmel N, Greenhalgh J, Manzano A, Monaghan M, Dalkin S: Doing realist research: Sage; 2018.

35. Marchal B, Kegels G, Belle SV: Theory and realist methods. In: Doing Realist Research. edn. Edited by Emmel N, Greenhalgh J, Manzano A, Monaghan M, Dalkin S. London: Sage; 2018: 79-91.

36. Pawson R: The science of evaluation: a realist manifesto: Sage; 2013.

37. Gilmore B, McAuliffe E, Power J, Vallières F: Data Analysis and Synthesis Within a Realist Evaluation: Toward More Transparent Methodological Approaches. International Journal of Qualitative Methods 2019, 18:1609406919859754.

38. Westhorp G: Realist impact evaluation: an introduction. London: Overseas Development Institute 2014:1-12.

39. Dalkin SM, Greenhalgh J, Jones D, Cunningham B, Lhussier M: What's in a mechanism? Development of a key concept in realist evaluation. Implementation Science 2015, 10(1):49.

40. Pawson R: Realist methodology: the building blocks of evidence. In: Evidence-based policy: a realist perspective edn. London: Sage; 2006: 17-38.

41. Schoonenboom J, Johnson RB: How to construct a mixed methods research design. KZfSS Kölner Zeitschrift für Soziologie und Sozialpsychologie 2017, 69(2):107-131. 
42. Morse JM: Principles of mixed methods and multimethod research design. Handbook of mixed methods in social and behavioral research 2003, 1:189-208.

43. Johnson RB, Onwuegbuzie AJ, Turner LA: Toward a definition of mixed methods research. Journal of mixed methods research 2007, 1(2):112-133.

44. Ivankova NV, Creswell JW, Stick SL: Using mixed-methods sequential explanatory design: From theory to practice. Field methods 2006, 18(1):3-20.

45. Malterud K: Utvalg [Norwegian] [Sample]. In: Kvalitative metoder I medisinsk forskningsmetoder for medisin og helsefag [in Norwegian][Qualitative Methods in Medical Research] 4th edn. Oslo, Norway: Universitetsforlaget; 2018: 57-67.

46. Naderifar M, Goli H, Ghaljaie F: Snowball sampling: A purposeful method of sampling in qualitative research. Strides in Development of Medical Education 2017, 14(3).

47. Manzano A: The craft of interviewing in realist evaluation. Evaluation 2016, 22(3):342-360.

48. Hsieh H-F, Shannon SE: Three Approaches to Qualitative Content Analysis. Qualitative Health Research 2005, 15(9):1277-1288.

49. International QSR: NVivo qualitative data analysis software 12 Pro. In.: QSR International Melbourne, Australia; 2019.

50. Watt RG, Daly B, Allison P, Macpherson LM, Venturelli R, Listl S, Weyant RJ, Mathur MR, GuarnizoHerreño CC, Celeste RK: Ending the neglect of global oral health: time for radical action. The Lancet 2019, 394(10194):261-272.

51. Martin SA, Simon L: Oral Health and Medicine Integration: Overcoming Historical Artifact to Relieve Suffering. American journal of public health 2017, 107(S1):S30-S31.

52. Lutfiyya MN, Gross AJ, Soffe B, Lipsky MS: Dental care utilization: examining the associations between health services deficits and not having a dental visit in past 12 months. BMC Public Health 2019, 19(1):265.

53. Petersen PE, Bourgeois D, Ogawa H, Estupinan-Day S, Ndiaye C: The global burden of oral diseases and risks to oral health. Bulletin of the World Health Organization 2005, 83:661-669.

54. Riggs E, Gussy M, Gibbs L, van Gemert C, Waters E, Kilpatrick N: Hard to reach communities or hard to access services? Migrant mothers' experiences of dental services. Aust Dent J 2014, 59(2):201207.

55. Leake JL, Birch S: Public policy and the market for dental services. Community Dentistry and Oral Epidemiology 2008, 36(4):287-295.

56. Meyerhoefer CD, Zuvekas SH, Farkhad BF, Moeller JF, Manski RJ: The demand for preventive and restorative dental services among older adults. Health Econ 2019, 28(9):1151-1158.

57. Gorbova M, John TM: Taking a bite out of policies: a look at the policies affecting our nation's dental health. Journal of Pediatric Nursing 2004, 19(1):51.

58. Halvorsen B, Willumsen T: Willingness to pay for dental fear treatment. The European Journal of Health Economics 2004, 5(4):299-308. 
59. Gulliford M, Figueroa-Munoz J, Morgan M, Hughes D, Gibson B, Beech R, Hudson M: What does' access to health care'mean? Journal of health services research \& policy 2002, 7(3):186-188.

60. Lundberg L, Johannesson M, Isacson DG, Borgquist L: Effects of user charges on the use of prescription medicines in different socio-economic groups. Health Policy 1998, 44(2):123-134.

61. Glick M, Williams DM, Kleinman DV, Vujicic M, Watt RG, Weyant RJ: A new definition for oral health developed by the FDI World Dental Federation opens the door to a universal definition of oral health. Journal of Public Health Dentistry 2017, 77(1):3-5.

62. Norwegian Directorate of Health: God klinisk praksis i tannhelsetjenesten (Good clinical practice in dental health). In. Edited by Directorate) HH. Oslo: Helsedirektoratet (Health Directorate); 2011.

63. Bastos LF, Hugo FN, Hilgert JB, Cardozo DD, Bulgarelli AF, Santos CMD: Access to dental services and oral health-related quality of life in the context of primary health care. Braz Oral Res 2019, 33:e018.

64. Mehrstedt M, John MT, Tonnies S, Micheelis W: Oral health-related quality of life in patients with dental anxiety. Community Dentistry and Oral Epidemiology 2007, 35(5):357.

65. Svensson L, Hakeberg M, Wide U: Dental pain and oral health-related quality of life in individuals with severe dental anxiety. Acta Odontologica Scandinavica 2018, 76(6):401-406.

66. McDermott AM, Fitzgerald L, Buchanan DA: Beyond acceptance and resistance: entrepreneurial change agency responses in policy implementation. British Journal of Management 2013, 24:S93S115.

67. Schofield J: Time for a revival? Public policy implementation: a review of the literature and an agenda for future research. International Journal of Management Reviews 2001, 3(3).

68. Lipsky M: Street-level bureaucracy: Dilemmas of the individual in public service: Russell Sage Foundation; 2010.

69. Akers HF, Foley MA, Brown JP, Woodford V: Public Dental Services, Queensland: Alfred James Hoole. $J$ Hist Dent 2018, 66(2):81-96.

70. McDermott AM, Hamel LM, Steel D, Flood PC, Mkee L: Hybrid healthcare governance for improvement? Combining Top-Down and Bottom-Up Approaches to Public Sector Regulation. Public Administration 2015, 93(2):324-344.

71. Mannion R, Davies $\mathrm{H}$ : Understanding organisational culture for healthcare quality improvement. $B M J$ 2018, 363:k4907.

72. Pfadenhauer LM, Gerhardus A, Mozygemba K, Lysdahl KB, Booth A, Hofmann B, Wahlster P, Polus S, Burns J, Brereton L: Making sense of complexity in context and implementation: the Context and Implementation of Complex Interventions (CICl) framework. Implementation science 2017, 12(1):21.

73. Davidson N, Skull S, Calache H, Chesters D, Chalmers J: Equitable access to dental care for an at-risk group: a review of services for Australian refugees. Australian and New Zealand Journal of Public Health 2007, 31(1):73-80. 


\section{Figures}

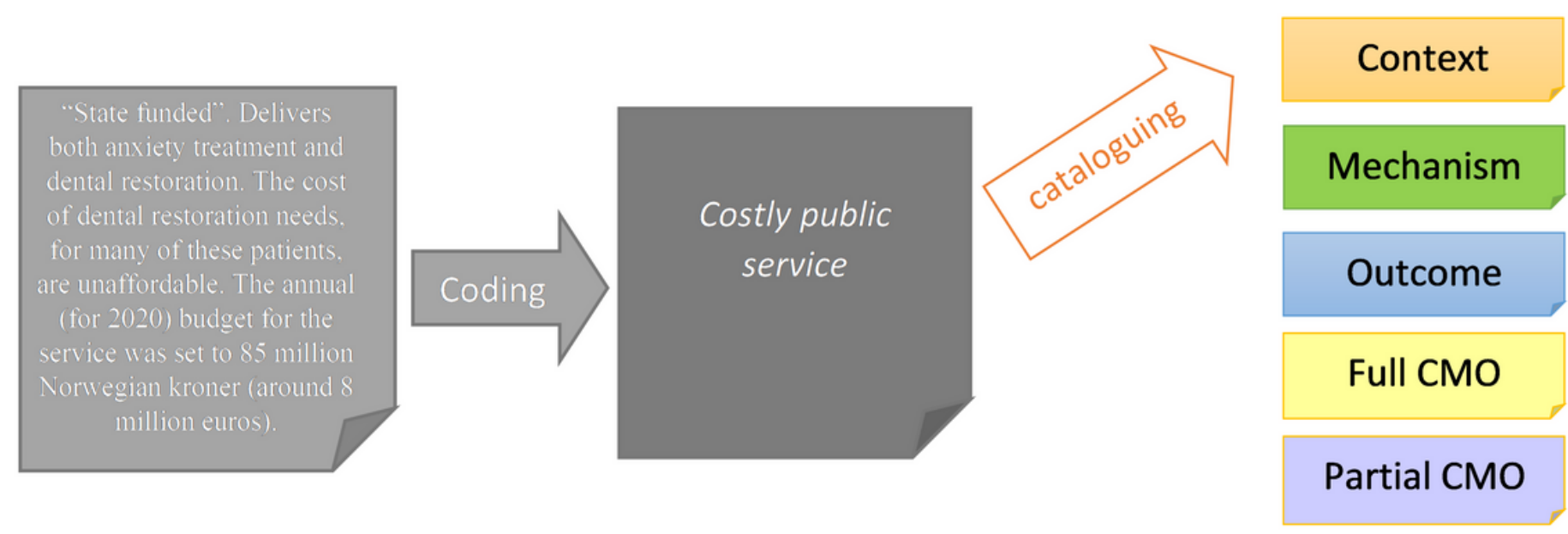

Figure 1

Steps of coding and cataloguing.

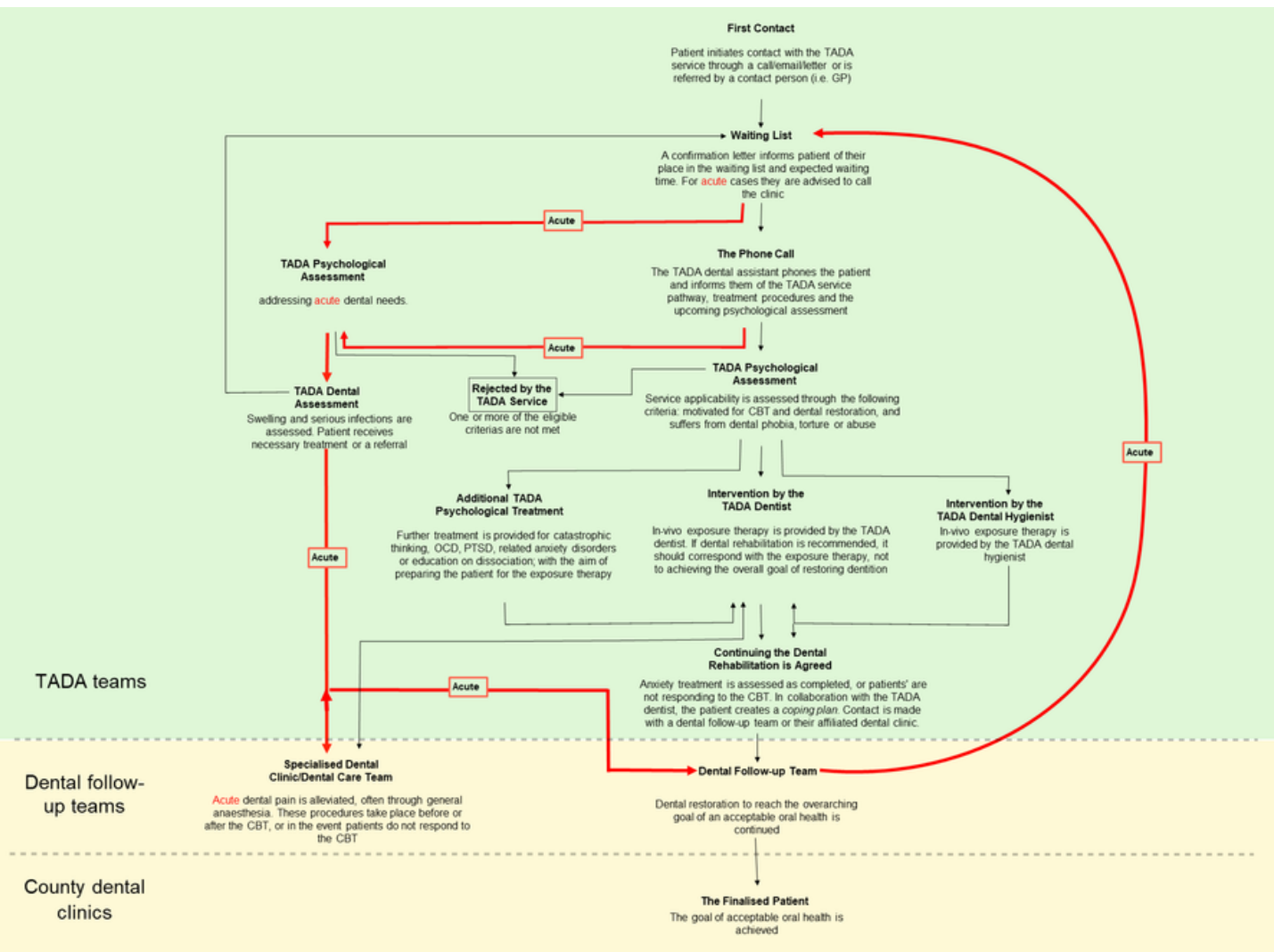

Figure 2

The TADA Service Pathway.

\section{Supplementary Files}


This is a list of supplementary files associated with this preprint. Click to download.

- ISSMCOREQChecklist.pdf 